\title{
Influx of Kininogens into Nasal Secretions after Antigen Challenge of Allergic Individuals
}

Claus R. Baumgarten, Alkis G. Togias, Robert M. Naclerio, Lawrence M. Lichtenstein, Philip S. Norman, and David Proud Division of Clinical Immunology, Departments of Medicine and Otolaryngology, The Johns Hopkins University School of Medicine at The Good Samaritan Hospital, Baltimore, Maryland 21239

\begin{abstract}
We have recently demonstrated that kinins are generated in vivo after nasal challenge with antigen of allergic, but not nonallergic, individuals. The present study was undertaken as a first step in determining the mechanism(s) of kinin formation during the allergic reaction and was directed towards establishing the availability and origin of kininogens in nasal secretions. Allergic individuals $(n=6)$ and nonallergic controls $(n=5)$ were challenged with antigen; and by using specific radioimmunoassays, nasal washes, obtained before and after challenge, were assayed for high molecular weight kininogen (HMWK), total kininogen (TK), albumin, and kinins. Dramatic increases in HMWK $(1,730 \pm 510 \mathrm{ng} / \mathrm{ml})$, TK $(3,810 \pm 1035 \mathrm{ng} / \mathrm{ml})$, kinin $(9.46 \pm 1.75 \mathrm{ng} / \mathrm{ml})$, and albumin $(0.85 \pm 0.2 \mathrm{mg} / \mathrm{ml})$ were observed after challenge of allergic individuals which correlated $(P<0.001)$ with increases in histamine and $N$ - $\alpha$-tosyl-Larginine methyl esterase activity and with the onset of clinical symptoms. For nonallergic individuals, levels of kininogens, albumin, and all mediators after antigen challenge were not different from base line. Linear regression analysis revealed excellent correlations $(P<0.001$ in each case) between increases in HMWK, TK, kinin, and albumin during antigen titration experiments and between the time courses of appearance and disappearance of HMWK, TK, kinin, and albumin after antigen challenge. Gel filtration revealed no evidence of degradation products of kininogens in nasal washes. For each allergic individual the ratio of HMWK/TK in postchallenge nasal washes was similar to the ratio of these two proteins in the same individual's plasma. These data suggest that, during the allergic reaction, there is an increase in vascular permeability and a transudation of kininogens from plasma into nasal secretions, where they can provide substrate for kinin-forming enzymes.
\end{abstract}

\section{Introduction}

Using a model of nasal challenge, we have recently provided the first direct evidence that kinins are generated during a local allergic reaction (1). The levels of kinins detected in nasal lavages during the allergic response are sufficient to cause relatively profound physiologic effects and suggest that these

This is Publication No. 599 of the O'Neill Research Laboratories, The Good Samaritan Hospital, Baltimore, MD.

Address reprint requests to Dr. Baumgarten.

Received for publication 13 August 1984 and in revised form 28 February 1985.

J. Clin. Invest.

(C) The American Society for Clinical Investigation, Inc.

0021-9738/85/07/0191/07 \$1.00

Volume 76, July 1985, 191-197 peptides may play a role in the pathogenesis of the allergic reaction. As yet, no specific kinin antagonist has been found. To evaluate definitively the extent to which kinins may contribute to the symptomatology of the allergic response, therefore, it will be necessary to reduce specifically or to abolish kinin formation during allergic reactions; thus, it is obviously essential to establish the mechanisms by which kinins are formed in response to antigen challenge. In that both bradykinin and lysylbradykinin are produced during the nasal response to allergen, the presence and involvement of several enzymes, including glandular kallikrein $(2,3)$, plasma kallikrein $(4,5)$, mast cell kininogenase (6), and kinin-converting aminopeptidase (7) must eventually be examined. An additional important factor that can regulate kinin formation, however, is the availability of substrate. Indeed, in the kidney, it has been suggested that kininogen availability may be the rate-limiting step in kinin formation (8). As a first step in understanding kinin formation during the nasal response to allergen, we decided, therefore, to examine the availability and origin of kininogens in nasal secretions. To determine whether kininogens are produced locally in the nasal mucosa, or whether high molecular weight kininogen (HMWK) ${ }^{1}$ and/or low molecular weight kininogen (LMWK) are capable of diffusing into nasal secretions from plasma, we utilized specific radioimmunoassays for HMWK (9) and total kininogen (TK) (10), as well as for human serum albumin (as a marker of plasma influx) to examine the concentrations of these proteins in nasal lavages during antigen titration experiments and time course studies and to relate the levels of each protein to the concentration of kinins and mast cell-derived mediators.

\section{Methods}

\section{Subjects}

Allergic individuals $(n=6)$ and nonallergic controls $(n=5)$ between the ages of 21 and $45 \mathrm{yr}$ were recruited. They gave informed consent before being included in the study. Allergic individuals were defined as having seasonal symptoms to an antigen to which they had a positive intradermal skin reaction at a concentration of 10 protein nitrogen units (PNU)/ml or less of crude allergen extracts. Allergic individuals were challenged "out of season" and were all asymptomatic at the time of challenge. Nonallergic individuals had no allergic history and negative skin tests to antigen extracts at a concentration of 100 $\mathrm{PNU} / \mathrm{ml}$.

\section{Nasal challenge and sample collection}

Ragweed extract and mixed grass extract (Greer Laboratories, Lenoir, NC) were used as allergens. The technique of nasal challenge with

1. Abbreviations used in this paper: HMWK, high molecular weight kininogen; HSA, human serum albumin; LMWK, low molecular weight kininogen; PNU, protein nitrogen unit; TAME, $N$ - $\alpha$-tosyl-Larginine methyl ester, TK, total kininogen. 
antigen has been described $(1,11)$. In brief, antigen extracts were either insufflated into the nose by use of a modified DeVilbiss 646 nebulizer (DeVilbiss Co., Somerset, PA) attached to a Rosenthal-French dosimeter (Laboratory of Applied Immunology, Baltimore, MD) or administered by instillation of $0.1 \mathrm{ml}$ of extract from a tuberculin syringe. Doses of antigen used for challenge ranged from 100 to 2000 PNU. (Although allergic responses can be obtained in our system with considerably less antigen, these higher doses were used to accentuate results for experimental purposes.) Nonallergic controls were always challenged with the highest doses of antigen used for allergics (i.e., 500, 1,000, and 2,000 PNU). Nasal secretions were collected by a 10-ml lavage with saline. In the absence of antigen challenge, repetitive saline lavages do not induce mediator production. Two different challenge protocols were used. Each protocol involved four prewashes to reduce cell-free mediator concentrations to a stable base line. Oxymetazoline hydrochloride (Afrin, Schering Corp., Kenilworth, NJ) was then administered to reduce mucosal edema, which occurs in allergics in response to antigen and interferes with lavage. (We have previously shown [11] that administration of oxymetazoline does not affect mediator production in response to a subsequent antigen challenge. In addition, although oxymetazoline may, on isolated occasions, induce one sneeze in a patient, we have not observed significant mediator production in saline lavages after administration of oxymetazoline.) $10 \mathrm{~min}$ after oxymetazoline administration, an additional lavage was performed. At this stage, the two protocols differ. In antigen titration (dose-response) experiments, two control challenges with the diluent used as a vehicle for the antigen extract (phenol-buffered saline, pH 7.4) were followed by three challenges with increasing doses of antigen. Lavages were performed $10 \mathrm{~min}$ after each control and antigen challenge. In time course experiments, a single control challenge was followed, after 10 min, by a lavage. A single antigen challenge was then given, and lavages were performed every $2 \mathrm{~min}$ for a period of $20 \mathrm{~min}$, beginning 2-4 min after challenge (to allow time for sneezing to subside). In all challenge experiments, nasal lavages were immediately made $40 \mathrm{mM}$ in EDTA and stored on ice until the completion of the experiment. The sol and mucus phases were then separated by centrifugation at $15,000 \mathrm{~g}$ for $15 \mathrm{~min}$ at $4^{\circ} \mathrm{C}$, and the sol phase was aliquoted and stored at $-80^{\circ} \mathrm{C}$.

\section{Assay methods}

Histamine, kinins, and enzymes displaying activity against $\left[{ }^{3} \mathrm{H}\right] \mathrm{N}-\alpha-$ tosyl-L-arginine methyl ester (TAME) were measured as previously described $(1,11)$. In brief, $0.8-\mathrm{ml}$ aliquots for histamine analysis were mixed with $0.2 \mathrm{ml}$ of $8 \% \mathrm{HClO}_{4}$ and centrifuged and the supernatants were assayed by an automated fluorometric technique capable of detecting $1 \mathrm{ng} / \mathrm{ml}$ of histamine (12). Enzyme(s) displaying arginine esterase activity were assayed by the radiochemical assay of Imanari et al. (13), which is based on the liberation of ${ }^{3} \mathrm{H}$-labeled methanol from the synthetic substrate $\left[{ }^{3} \mathrm{H}\right] \mathrm{TAME}$. Kinins are assayed by using a specific radioimmunoassay capable of detecting $20 \mathrm{pg} / \mathrm{ml}$ of kinin (1).

Kininogens. The specific radioimmunoassays for human TK (10) and HMWK (9) have been described previously. The assay for TK utilizes an antibody that recognizes the heavy chain common to both HMWK and LMWK. Highly purified human LMWK was used as a standard and values for unknowns were expressed relative to this standard. The assay for HMWK was an antibody that recognizes the unique light chain of HMWK, and high purified HMWK is used as a standard. Results from the HMWK assay allowed calculations of the contribution of HMWK to TK values, which were then corrected to take into account the molecular weight differences between the two kininogen molecules. For example, if a sample gave an apparent TK content of $3 \mu \mathrm{g} / \mathrm{ml}$ in the TK assay, although the same sample contained $1 \mu \mathrm{g} / \mathrm{ml}$ HMWK as measured in the HMWK assay, the TK value would be corrected as follows (assuming molecular weights of 110,000 and 68,000 for HMWK and LMWK, respectively): $1 \mu \mathrm{g} / \mathrm{ml}$ HMWK would be detected as $1 \times 68,000 / 110,000=0.62 \mu \mathrm{g} / \mathrm{ml}$ in the TK assay. Hence, of the $3 \mu \mathrm{g} / \mathrm{ml}$ measured in the TK assay, $3-0.62=2.38 \mu \mathrm{g} / \mathrm{ml}$ would represent LMWK. A true estimate of
TK, therefore, is $2.38 \mu \mathrm{g} / \mathrm{ml} \mathrm{LMWK}+1 \mu \mathrm{g} / \mathrm{ml} \mathrm{HMWK}=3.38 \mu \mathrm{g} /$ ml TK.

The highly purified human kininogens used for iodination and for standards were prepared by a minor modification of the method of Pierce and Guimaraes (14) that utilized batch adsorption with DEAESephacel, affinity chromatography on lysine-agarose to remove plasmin and plasminogen, immunoaffinity chromatography, and DEAE-Sephacel chromatography to separate HMWK and LMWK by means of a phosphate gradient. When nasal washes were examined in each assay, inter- and intraassay coefficients of variation (standard deviation per $x$ $\times 100 \%)$ were $10.7 \%(n=6)$ and $3 \%(n=7)$, respectively, for TK and $15.1 \%(n=5)$ and $6.5 \%(n=13)$, respectively, for HMWK.

Human serum albumin. Human serum albumin (HSA) was measured by radioimmunoassay. Antiserum to highly purified human albumin was produced in a sheep and antibody was purified by affinity chromatography using purified HSA immobilized on Sepharose 4B. This antibody, which was a generous gift of Dr. Jack V. Pierce (National Heart, Lung and Blood Institute, Bethesda, MD), showed a single arc on immunodiffusion against whole human serum, corresponding to that produced by purified HSA. Bovine serum albumin showed little or no cross-reaction at concentrations up to $10 \mathrm{mg} / \mathrm{ml}$. Highly purified HSA was iodinated by the method of Hunter and Greenwood (15). A $70-\mu$ l volume $(10 \mu \mathrm{g})$ of HSA in $0.1 \mathrm{M}$ Tris/ $0.025 \% \mathrm{NaN}_{3}, \mathrm{pH} 7.4$, was mixed with $5 \mu \mathrm{l}(0.5 \mathrm{mCi})$ of $\mathrm{Na}^{125} \mathrm{I}$ (Amersham Corp., Arlington Heights, IL). The reaction was initiated by addition of $25 \mu \mathrm{l}(50 \mu \mathrm{g})$ of an aqueous solution of chloramine T and was allowed to proceed for $2 \mathrm{~min}$ on ice until termination by the addition of $25 \mu \mathrm{l}(500 \mu \mathrm{g}) \mathrm{Na}_{2} \mathrm{~S}_{2} \mathrm{O}_{5}$. After the addition of $25 \mu \mathrm{l}(500$ $\mu \mathrm{g}$ ) of potassium iodide, labeled protein was separated from free iodide by two successive gel filtrations on a column $(0.8 \times 16 \mathrm{~cm})$ of Sephadex G-75 (Pharmacia Fine Chemicals, Piscataway, NJ) equilibrated with $0.1 \mathrm{M}$ Tris $/ 0.025 \% \mathrm{NaN}_{3} / 0.1 \%$ bovine albumin, $\mathrm{pH}$ 7.4. The labeled HSA was frozen and stored in aliquots at $-80^{\circ} \mathrm{C}$.

Assays were performed in $0.1 \mathrm{M}$ Tris $/ 0.025 \% \mathrm{NaN}_{3} / 0.1 \%$ bovine serum albumin, $\mathrm{pH} 7.4$, and all dilutions were made in this buffer. A $200-\mu 1$ volume of standard or unknown, at a suitable dilution, was incubated with $100 \mu \mathrm{l}$ of labeled HSA $(9,000 \mathrm{cpm})$ and $100 \mu \mathrm{l}$ of antibody $\left(1: 400,000\right.$ final dilution) for $20-24 \mathrm{~h}$ at $4^{\circ} \mathrm{C}$. Antigenantibody complex was separated from free antigen by immunoprecipitation as follows. A $10-\mu \mathrm{l}$ portion of a $0.5 \mathrm{mg} / \mathrm{ml}$ solution of sheep IgG followed by $300 \mu \mathrm{l}$ of a 1:20 dilution of rabbit antisheep IgG (Cappel Scientific, Malvern, PA) were added to each tube and all samples incubated for $2 \mathrm{~h}$ at room temperature. Then $2 \mathrm{ml}$ of buffer was added to each tube, and antigen-antibody complex was sedimented by centrifugation at $1,500 \mathrm{~g}$ for $10 \mathrm{~min}$. The supernatants were removed to a fixed mark by aspiration. The buffer addition, centrifugation, and aspiration procedures were repeated and the radioactivity associated with the precipitate was counted. Results were calculated with a Spline-fit computer program.

\section{Statistical analysis}

Correlations between absolute increases in mediator levels were calculated by linear regression analysis and by stepwise regression analysis using the Biomedical Data Processing package on the IBM 4341 computer (IBM Corp., Danbury, CT) (16). Significance of the elevation of the mean level of each mediator in the allergic compared to nonallergic group was determined by a Mann-Whitney $U$ test.

\section{Results}

Representative results obtained upon nasal challenge of an allergic individual by using the antigen titration protocol are shown in Fig. 1. Low but detectable levels of HMWK, TK, and albumin were present in washes obtained during the baseline phase of the experiment. After antigen challenge, the dramatic, dose-dependent increase in kinin was paralleled by increases in HMWK, TK, and albumin. As previously reported 


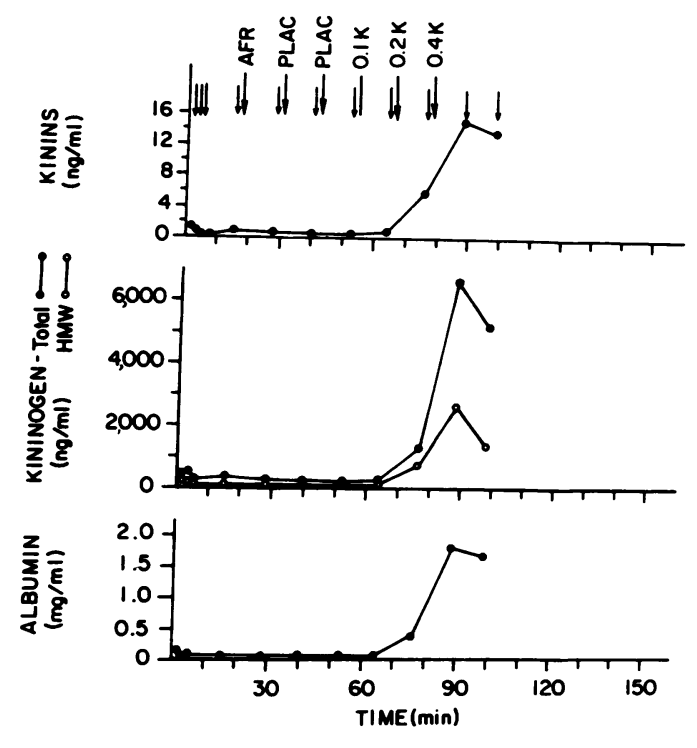

Figure 1. Nasal challenge of an allergic individual by use of the antigen titration protocol. The experimental protocol is shown at the top of the figure. The smaller arrow indicates saline wash and the larger arrow indicates administration of the material indicated. Pollen extract dosages are in thousands of protein nitrogen units per milliliter (K). A 0.1-ml sample of extract was administered at each dose. Kinin, kininogen, and albumin increases were also accompanied by increases in histamine and TAME-esterase activity and by the onset of clinical symptoms (not shown). AFR, Afrin (oxymetazoline hydrochloride); PLAC, placebo.

(1), the increase in kinin levels also correlated with increases in histamine and TAME-esterase activity and with the onset of clinical symptoms (not shown).

To ensure that quantitative measurements of kininogens could be made during comparisons of allergic and nonallergic individuals, serial dilutions of nasal lavages were tested in each assay. As can be seen in Fig. 2, dilutions of nasal washes produced a displacement curve which paralleled the standard curve in the assay for TK. Similar results were also obtained

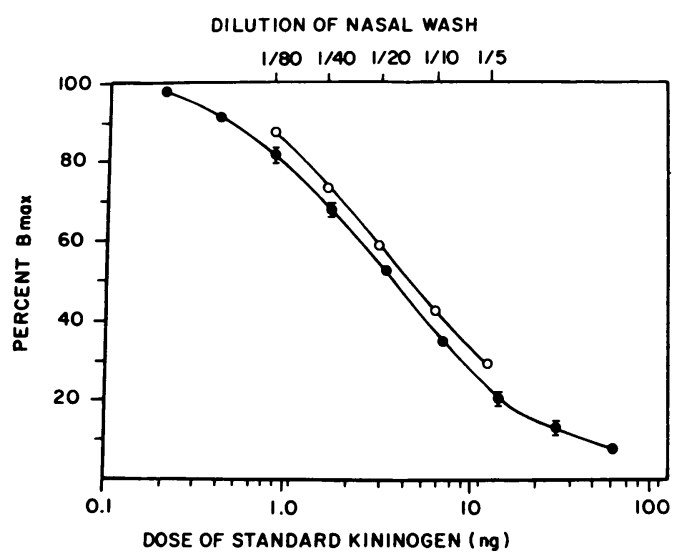

Figure 2. Parallel cross-reaction of nasal washes in the radioimmunoassay for total kininogen. Serial dilutions of a postchallenge nasal wash containing immunoreactive kininogen $\left(-O_{-}\right)$were compared with the standard curve $(-\bullet-) . B_{\max }$, maximum binding in the absence of nonlabeled antigen.

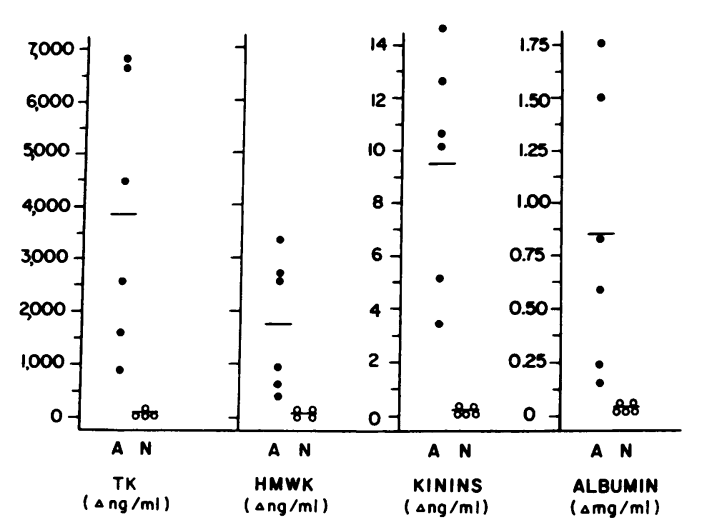

Figure 3. A comparison of the concentrations of kinins, kininogens, and albumin in nasal washes after challenge of allergic $(A, \bullet)$ and nonallergic $(N, O)$ individuals. The dots represent the maximum concentration of kinin or each protein in nasal washes of each individual after challenge. The arithmetic means for each parameter are represented by horizontal lines. TK, $P<0.005$; HMWK, $P<0.005$; kinins, $P<0.002$; albumin, $P<0.002$.

in the assays for HMWK and albumin. Recovery of standard kininogens added to nasal washes was $92 \pm 2 \%$ ( $\bar{x} \pm$ SEM; $n=9)$ for TK and $111 \pm 4 \%(n=8)$ for HMWK. Recovery did not vary significantly between control and allergic individuals, nor was there any difference in recovery of standard kininogens from prechallenge vs. postchallenge nasal washes.

When allergics and nonallergics were compared in antigen titration experiments, the results shown in Fig. 3 were obtained. For nonallergics, levels of kinin, HMWK, TK, and albumin were not different from base line. Challenge of allergics, however, resulted in highly significant increases in the concentrations of kinin $(9.46 \pm 1.75 \mathrm{ng} / \mathrm{ml})$, HMWK $(1730 \pm 510 \mathrm{ng} /$ $\mathrm{ml})$, TK $(3810 \pm 1035 \mathrm{ng} / \mathrm{ml})$, and albumin $(0.85 \pm 0.2 \mathrm{mg} / \mathrm{ml})$ in nasal lavages ( $\bar{x} \pm \mathrm{SEM}$ in each case). Significant increases $(P<0.002$ in each case $)$ were also seen in levels of histamine and TAME-esterase activity (not shown). It should be noted that, at all points in all titration experiments, levels of kininogen were more than adequate to account for the levels of kinin detected.

Linear regression analysis of mediator and protein levels in all postchallenge washes from the antigen titration experiments of the six allergics revealed excellent correlations between the levels of all mediators and proteins (Table I). Examination of the data by stepwise regression analysis with TK as the

Table I. Correlations* between the Concentrations of Mediators, Kininogens, and Albumin in Nasal Lavages during Antigen Titration Experiments (Linear Regression Analysis)

\begin{tabular}{llllll}
\hline & TK & HMWK & Kinin & $\begin{array}{c}\text { TAME- } \\
\text { esterase }\end{array}$ & Histamine \\
& $r$ & $r$ & $r$ & $r$ & $r$ \\
HMWK & 0.94 & & & & \\
Kinin & 0.79 & 0.75 & & & \\
TAME-esterase & 0.81 & 0.85 & 0.66 & & \\
Histamine & 0.83 & 0.81 & 0.68 & 0.76 & \\
Albumin & 0.89 & 0.83 & 0.73 & 0.80 & 0.72 \\
& & & & & \\
\hline
\end{tabular}

$* P<0.001$ in all cases. 
dependent variable revealed, not unexpectedly, that HMWK was the first independent variable selected to explain the variations in TK $(r=0.938)$. The second step of the regression, however, resulted in an increase in the correlation coefficient (to $r=0.960$ ) by selection of albumin as the second variable. No other variables were selected as significant.

There has been no report on the presence of HMWK other than in plasma or, in lower amounts, in platelets (17). Thus, the influx of HMWK into nasal secretions during the allergic response, together with the quantitative correlations between HMWK, TK, and albumin, would suggest that the increases in nasal kininogens may be due to plasma influx. Support for this concept comes from a comparison of the ratios of HMWK/ TK in nasal secretions vs. plasma for each of the allergic individuals challenged (Table II). By Wilcoxon matched-pair, signed-rank analysis, no significant difference was found between plasma values and values in nasal secretions.

If the increases in kininogens in nasal secretions are indeed due to plasma influx, then a temporal relationship should also exist between the levels of HMWK, TK, and albumin after antigen challenge. To examine this possibility, three allergic individuals were each challenged with a single dose of antigen, according to the protocol described in Methods. An example of the results obtained is shown in Fig. 4. After antigen challenge, the appearance and disappearance of histamine, TAME-esterase activity, kinins, HMWK, TK, and albumin all seemed to follow a constant temporal relationship. The meaning of the second peak of mediators and proteins in Fig. 4 is not clear, but a similar peak occurred with the other two patients. Linear regression analysis of all positive points from the three time course experiments revealed excellent correlation coefficients between all mediators and proteins (Table III). In stepwise regression analysis of these data with TK as the dependent variable, the variation in TK was, again, best explained by HMWK $(r=0.90)$, with albumin levels also being a significant predictor of $\mathrm{TK}$, increasing the correlation coefficient to 0.95 . Thus, as for the dose-response data, HMWK, TK, and albumin are all highly correlated.

Additional evidence to support the plasma origins of kininogens in nasal lavages was obtained from gel filtration studies. Chromatography of concentrated nasal washes on Sephacryl S-200 in high-salt buffer (Fig. 5) revealed profiles for HMWK and TK similar to those observed if plasma is chromatographed under the same conditions of high ionic

Table II. Comparison of Ratio of HMWK/TK in Nasal Washes and Plasma of Allergic Individuals

\begin{tabular}{lllll}
\hline Patient & Plasma ratio* & $n \ddagger$ & Nasal wash ratio* & $n \ddagger$ \\
\hline & $\%$ & & $\%$ & \\
1 & 45 & 1 & $50 \pm 8.5$ & 4 \\
2 & 46 & 1 & $36 \pm 0.3$ & 2 \\
3 & $45 \pm 6.8$ & 3 & $41 \pm 8.7$ & 4 \\
4 & $51 \pm 4.5$ & 3 & $41 \pm 14$ & 3 \\
5 & $63 \pm 11$ & 2 & $66 \pm 3.4$ & 3 \\
6 & 53 & 1 & $50 \pm 7.5$ & 4 \\
\hline
\end{tabular}

* Expressed as mean $\pm \mathrm{SD}$.

$\ddagger n$, number of determinations.
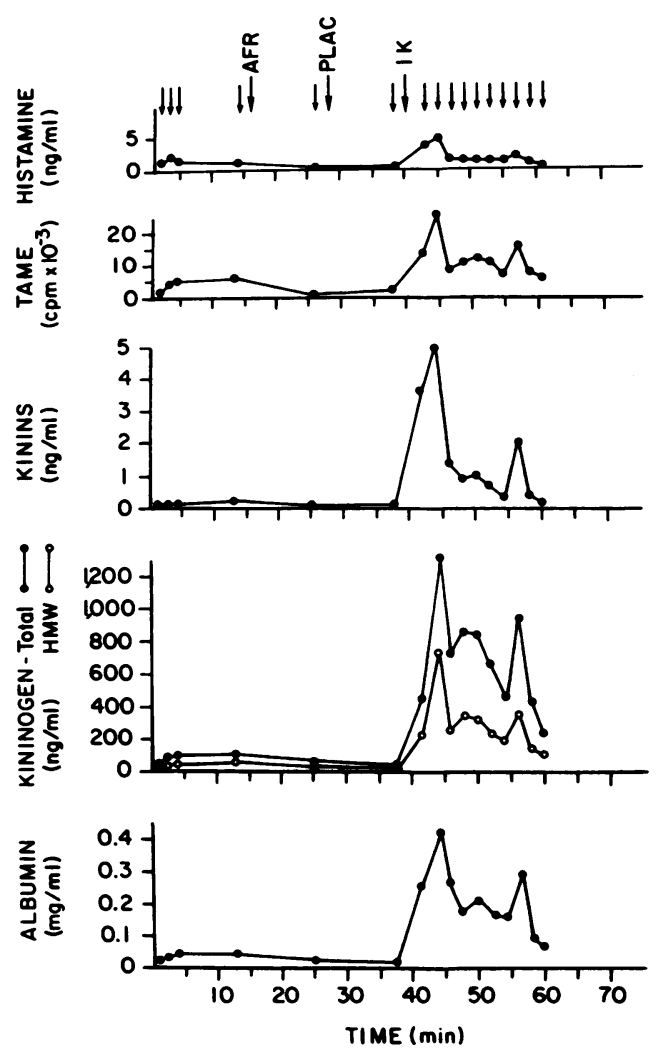

Figure 4. Time course of appearance and disappearance of mediators and proteins after antigen challenge. The experimental protocol, involving a single dose challenge, is shown at the top of the figure. Symbols and abbreviations are as described for Fig. 1.

strength (not shown). There was no evidence of low molecular weight degradation products of kininogens in any of three such experiments performed with nasal lavages.

\section{Discussion}

In recent years, the list of inflammatory mediators implicated in the allergic reaction has increased considerably. The pharmacologic actions of kinins have led several investigators to suggest that they may also play a role in the allergic response

Table III. Correlations* between the Concentration of Mediators, Kininogens, and Albumin in Nasal Lavages during Time Course Experiments (Linear Regression Analysis)

\begin{tabular}{llllll}
\hline & TK & HMWK & Kinin & $\begin{array}{r}\text { TAME- } \\
\text { esterase }\end{array}$ & Histamine \\
& $r$ & $r$ & $r$ & $r$ & $r$ \\
HMWK & 0.90 & & & & \\
Kinin & 0.79 & 0.77 & & & \\
TAME-esterase & 0.89 & 0.86 & 0.92 & & \\
Histamine & 0.70 & 0.69 & 0.82 & 0.75 & \\
Albumin & 0.89 & 0.79 & 0.89 & 0.88 & 0.75 \\
& & & & & \\
\hline
\end{tabular}

$* P<0.001$ in all cases. 


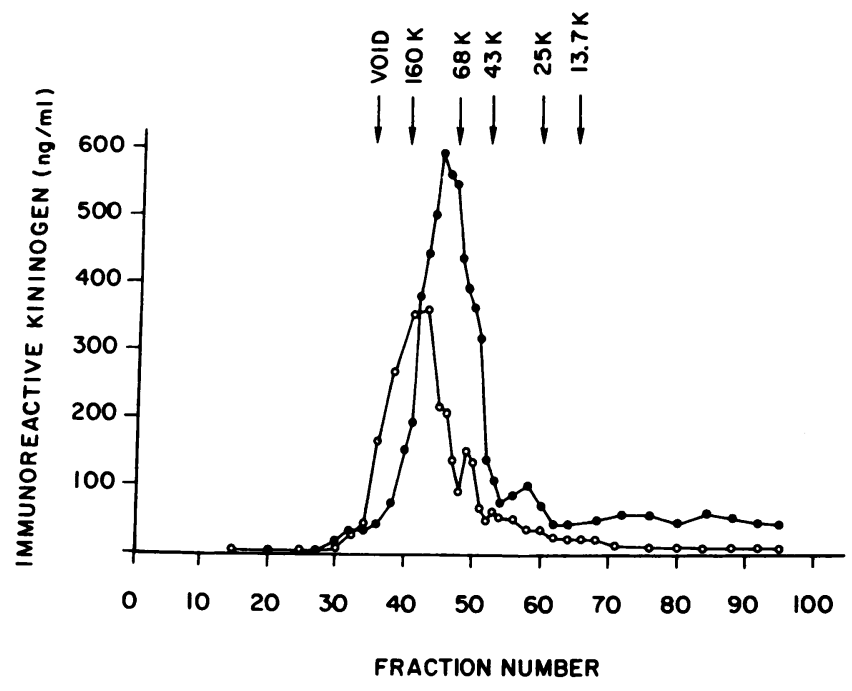

Figure 5. Sephacryl S-200 gel filtration of a nasal wash. A 1.5-ml sample of a postchallenge nasal wash containing immunoreactive kininogen was made $1 \mathrm{M}$ in $\mathrm{NaCl}$ and applied to a calibrated column $(1.6 \times 91 \mathrm{~cm})$ of Sephacryl S-200 equilibrated with $0.1 \mathrm{M}$ Tris/1.0 M NaCl, $\mathrm{pH}$ 7.4. The column was eluted at a flow rate of $12 \mathrm{ml} / \mathrm{h}$ and fractions of $2-\mathrm{ml}$ volume were collected. Fractions were then assayed using radioimmunoassays for HMWK (- -$)$, measuring the specific light chain of this molecule, and for TK (- $\bullet-)$, measuring the heavy chain common to both kininogens. The column was calibrated with the following standards: blue dextran, rabbit IgG, bovine serum albumin, ovalbumin, chymotrypsinogen, and ribonuclease $\mathrm{A}$.

(18, 19). Although indirect support for this hypothesis came from the observation that HMWK levels decreased during anaphylactic reactions in man (20), our recent studies showing that bradykinin and lysylbradykinin are generated after nasal antigen challenge of allergic, but not nonallergic, individuals provided the first direct evidence of kinin formation during a local allergic reaction (1). Although kinins have now been implicated in the allergic reaction, however, the mechanisms by which they may be generated in response to antigen challenge have yet to be delineated. The present study was undertaken as a first step towards elucidating these mechanisms and was directed towards establishing the availability, origin, and identity of substrate(s) for kinin-forming enzymes.

The major source of kininogens is plasma, where two forms, HMWK and low molecular weight kininogen (LMWK) are present. There is also precedent, however, for the existence of LMWK extravascularly. Thus, LMWK has been detected in human parotid and submaxillary salivas (21) and has been localized by immunofluorescence to the distal nephron of the kidney (10). To date, however, there has been no report of intact HMWK being detected in the absence of plasma.

Our present results show a clear increase in the concentrations of HMWK and TK (and, therefore, by difference, LMWK) in nasal lavages of allergic individuals after antigen challenge. The levels of kininogens at each point in every experiment were always more than sufficient to account for detected concentrations of kinin. These results could be explained if it were assumed that antigen, or mast cell mediators, stimulate kininogen production by nasal glands. Alternatively, kininogens may enter nasal secretions by transudation from plasma.
Increased influx of plasma proteins into nasal secretions is known to occur, for instance, in patients with nasal polyps (22) and in children with recurrent upper airway infections (23). Support for the plasma origin of the kininogens in nasal secretions is derived from the presence of intact HMWK, which to date has only been detected in plasma and platelets, and from the excellent correlations between the concentrations of kininogens and albumin. It should be noted that, while there are strong correlations between levels of all mediators and proteins using linear regression analysis, stepwise regression reveals that, in both dose-response and time course experiments, the best correlates with TK are HMWK and albumin. If kininogens were, indeed, diffusing into nasal secretions from plasma, then a fixed relationship between the levels of HMWK, TK, and albumin would be expected to occur in each type of nasal protocol. The regression analysis data are, therefore, consistent with this hypothesis. Further support for the concept of plasma influx is provided by the similar ratios of HMWK/ TK in nasal washes and plasma of allergic individuals, as well as by gel filtration data which reveals profiles for HMWK and TK which are essentially the same as those seen when plasma is gel-filtered under the same conditions of high ionic strength. The use of high-salt buffer (selected to prevent the adherence of proteins to the heparin proteoglycan from mast cells) results in the separation of HMWK from prekallikrein. It is interesting to note that, although mast cell mediators are clearly released during the allergic response, and while tryptase, the major neutral protease of human mast cells (24), has been reported to extensively degrade the light chain of HMWK in vitro (25), there is no evidence of immunoreactive degradation fragments upon gel filtration. Thus, although we cannot rule out the formation of some nonantigenic fragments of light chain, extensive generation of immunoreactive degradation products, such as those observed in urine (10), are not seen, suggesting that this tryptase-catalyzed reaction is not kinetically favorable in our present in vivo system.

The finding that ratios of HMWK/TK in nasal secretions and plasma are essentially the same indicates that both kininogens are able to diffuse into nasal secretions at the same rate. Because HMWK circulates in plasma as a complex with plasma prekallikrein (26) of mol wt 280,000 , this would suggest that the transudation of plasma proteins is not restricted to lower molecular weight molecules. In addition, because HMWK is the preferred substrate for plasma kallikrein $(14,27)$, the presence of this protein in nasal secretions would provide substrate for kinin formation by this enzyme should activation of prekallikrein occur during the allergic reaction.

We have previously shown that nasal lavage with $10 \mathrm{ml}$ of isotonic saline allows recovery of $\sim 8 \mathrm{ml}$ of nasal wash fluid (11). Mean values for postchallenge concentrations of HMWK, TK, and albumin in dose-response experiments indicate that, despite the prior administration of the vasoconstrictor, oxymetazoline, an 8-ml nasal wash contains, on average, the equivalent of $\sim 0.15-\mathrm{ml}$ plasma transudation. In some patients, levels of HMWK, TK, and albumin equivalent to those from $0.5 \mathrm{ml}$ of plasma were detected in nasal washes. It may be argued that differing distributions of antigen and oxymetazoline in the nose account for the vasodilatation and increased vascular permeability involved. We consider it more likely, however, that mast cell mediators are overriding, to some degree, the effects of oxymetazoline. Indeed, it should be noted that the main reason for the use of oxymetazoline in our 
experimental protocol was that, without the vasoconstrictor, nasal edema was so severe that almost complete obstruction of the nasal airways occurred, making lavage impossible. Although it must be admitted that the antigen challenges used in the present study may not adequately reflect field exposure, it is of interest that the levels of albumin detected in postchallenge washes of allergic individuals previously given oxymetazoline are very similar to those detected by Mygind and coworkers (28) in nasal secretions of untreated grass-allergic individuals during the course of an exceptionally mild grass pollen season. In contrast to this earlier study, however, our more controlled model allows us to show clearly significant differences between allergics and nonallergics.

Having shown that plasma proteins of high molecular weight (including HMWK and, presumably, prekallikrein) enter the nasal secretions as a result of antigen challenge, the question could arise as to whether the kinins measured during the allergic response may be generated, not as a direct consequence of the allergic reaction, but as a result of activation of the plasma kinin-generating system by the lavage process. It is possible, for instance, that after antigen challenge, the nasal mucosa may become so labile as to be traumatized by the lavage process, such that activation of the Hageman factordependent pathways could occur. Alternatively, it is known that the plasma kinin system can be activated by dilution in glass (29), so the dilution effects associated with lavage may result in kinin generation. Although it is not possible to totally exclude some activation of plasma kallikrein during the $10 \mathrm{~s}$ in which the saline is in the nose, we believe that the overwhelming majority of evidence to date indicates that kinins are formed in vivo as a part of the allergic response and are already present at the time of lavage. The concept of activation by dilution is unlikely, in that dilution of plasma in the plastic containers used during nasal challenge experiments does not result in measurable activation of plasma kallikrein even after $5 \mathrm{~h}$. If kinin formation occurred as a result of the lavage process, then additional kinin might be expected to be formed in the test tube after collection. In contrast, collection of aliquots of nasal lavages either into EDTA-containing benzamidine, a potent inhibitor of plasma kallikrein, or directly into $95 \%$ ethanol to precipitate proteins, yielded levels of kinins that were very similar to those found in aliquots of the same lavages collected in the usual manner. Thus, the high dilution factor associated with lavage may, indeed, help to prevent additional kinin formation. Finally, detection of lysylbradykinin in nasal lavages (1) cannot be explained by activation of the plasma kinin system, because plasma kallikrein generates only bradykinin (30). Other enzyme systems, for which activation by lavage would be hard to accept, must be involved. We conclude, therefore, that kinins are generated in vivo as a direct consequence of the allergic reaction, by mechanisms involving more than one enzyme, and that the kinins produced could contribute to the symptomatology of the allergic reaction.

In summary, we have shown that antigen challenge of allergic, but not nonallergic, individuals results in significant increases in the concentrations of HMWK, TK, and albumin in nasal lavages. The levels of these proteins are highly correlated with each other, as well as with mediator production during both dose-response and time course studies, and the measured concentrations of kininogens are always more than sufficient to account for the generated kinin. Although we cannot rule out the possibility that some small degree of local production of kininogens may occur, our findings are consistent with the hypothesis that, during the allergic reaction, mast cell mediators cause an increase in vascular permeability and an influx of kininogens into the nasal secretions, where they can provide substrate for kinin-forming enzymes.

\section{Acknowledgments}

The authors wish to thank Ms. Linda Friedhoff for help and useful discussion related to the statistical methods used and Mrs. Carol Dankelman for secretarial assistance.

This work was supported in part by grants HL-32272, NS-22488, AI-04866, AI-07290, and AI-20135 from the National Institutes of Health; a Johns Hopkins Institutional Research Grant (to Dr. Proud); and by grant BA656 from the Deutsche Forschungsgesellschaft, Federal Republic of Germany. Dr. Naclerio is the recipient of Teacher Development Investigator Award 1K07 NS-00811 from the National Institute of Neurological and Communicative Disorders and Stroke.

\section{References}

1. Proud, D., A. Togias, R. M. Naclerio, S. A. Crush, P. S. Norman, and L. M. Lichtenstein. 1983. Kinins are generated in vivo following nasal airway challenge of allergic individuals with allergen. J. Clin. Invest. 72:1678-1685.

2. Pisano, J. J. 1975. Chemistry and biology of the kallikrein-kinin system. In Proteases and Biological Control. E. Reich, D. B. Rifken, and E. Shaw, editors. Cold Spring Harbor Laboratory, Cold Spring Harbor, NY. 199-222.

3. Nustad, K., T. B. Orstavik, K. M. Gautvik, and J. V. Pierce. 1978. Glandular kallikreins. Gen. Pharmacol. 9:1-9.

4. Kaplan, A. P., M. Silverberg, J. T. Dunn, and B. Ghebrehiwet. 1982. Interaction of the clotting, kinin-forming, complement and fibrinolytic pathways in inflammation. Ann. NY Acad. Sci. 389:25-38.

5. Cochrane, C. G., and J. H. Griffin. 1982. The biochemistry and pathophysiology of the contact system of plasma. Adv. Immunol. 33: 241-306.

6. Proud, D., E. S. Schulman, D. W. MacGlashan, J. V. Pierce, and H. H. Newball. 1982. Anaphylactic release of a kininogenase from purified human lung mast cells. Clin. Res. 30:165A. (Abstr.)

7. Guimaraes, J. A., D. R. Borges, E. S. Prado, and J. L. Prado. 1973. Kinin-converting aminopeptidase from human serum. Biochem. Pharmacol. 22:3157-3172.

8. Pisano, J. J. 1983. Observations on the kallikrein-kinin system in the kidney. Adv. Exp. Med. Biol. 156:929-938.

9. Proud, D., J. V. Pierce, and J. J. Pisano. 1980. Radioimmunoassay of human high molecular weight kininogen in normal and deficient plasmas. J. Lab. Clin. Med. 95:563-574.

10. Proud, D., M. Perkins, J. V. Pierce, K. N. Yates, P. F. Highet, P. L. Herring, M. Mangkornkanok/Mark, R. Bahu, F. A. Carone, and J. J. Pisano. 1981. Characterization and localization of human renal kininogen. J. Biol. Chem. 256:10634-10639.

11. Naclerio, R. M., H. L. Meier, A. Kagey-Sobotka, N. F. Adkinson, Jr., D. A. Meyers, P. S. Norman, and L. M. Lichtenstein. 1983. Mediator release after nasal airway challenge with allergen. Am. Rev. Respir. Dis. 128:597-602.

12. Siraganian, R. 1971. An automated continuous flow system for the extraction and fluorometric analysis of histamine. Anal. Biochem. 57:283-294.

13. Imanari, T., T. Kaizu, H. Yoshida, K. Yates, J. V. Pierce, and J. J. Pisano. 1976. Radiochemical assays for human urinary, salivary and plasma kallikreins. In Chemistry and Biology of the KallikreinKinin System in Health and Disease. J. J. Pisano and K. F. Austen, editors. Department of Health, Education and Welfare Publ. No. (NIH) 76-791, Washington, DC. 205-213.

14. Pierce, J. V., and J. A. Guimaraes. 1976. Further characterization of highly purified human plasma kininogens. In Chemistry and Biology 
of the Kallikrein-Kinin System in Health and Disease. J. J. Pisano and K. F. Austen, editors. Department of Health, Education and Welfare Publ. No. (NIH) 76-791, Washington, DC. 121-127.

15. Hunter, W. M., and F. C. Greenwood. 1962. Preparation of ${ }^{131}$ I-labelled human growth hormone of high specific activity. Nature (Lond.). 194:495-496.

16. Dixon, W. J., and R. Jennrich. 1981. Stepwise regression. In BMDP Statistical Software 1981. W. J. Dixon, editor. University of California Press, Berkeley, CA. 251-263.

17. Schmaier, A. H., A. Zuckerburg, C. Silverman, J. Kuchibhotla, G. P. Tuszynski, and R. W. Colman. 1983. High-molecular weight kininogen. A secreted platelet protein. J. Clin. Invest. 71:1477-1489.

18. Spragg, J., R. C. Talamo, and K. F. Austen. 1970. Immunochemistry of bradykinin and immunologic activation of the kinin system. In Handbook of Experimental Pharmacology. Bradykinin, Kallidin and Kallikrein. Vol. 25. E. G. Erdos, editor. Springer-Verlag, Berlin, Heidelberg, New York. 531-549.

19. Brenner, M., C. Baumgarten, G. Kunkel, R. Rudolph, and E, Kirchhof. 1982. The possible role of bradykinin as a mediator of bronchial asthma. Allergol. Immunopathol. 10:453-462.

20. Smith, P. L., A. Kagey-Sobotka, E. R. Bleeker, R. Traystman, A. P. Kaplan, H. Gralnick, M. D. Valentine, S. Permutt, and L. M. Lichtenstein. 1980. Physiologic manifestations of human anaphylaxis. J. Clin. Invest. 66:1072-1080.

21. Sakamoto, W., H. Fukuda, and O. Nishikaze. 1981. Kininogen and kallikrein in saliva of periodontally-diseased subjects. J. Dent. Res. 60:6-9.
22. Sorensen, H., N. Mygind, C. B. Pedersen, and S. Prytz. 1976. Long-term treatment of nasal polyps with beclomethasone dipropionate aerosol. III. Morphological studies and conclusions. Acta Otolaryngol. 82:260-262.

23. Mygind, N., and J. A. Wihl. 1976. Concentrations of immunoglobulins in nasal secretion from children with recurrent infections in the upper airways. Acta Otolaryngol. 82:216-218.

24. Schwartz, L. B., R. A. Lewis, and K. F. Austen. 1981. Tryptase from human pulmonary mast cells. Purification and characterization. J. Biol. Chem. 256:11939-11943.

25. Maier, M., J. Spragg, and L. B. Schwartz. 1983. Inactivation of human high molecular weight kininogen by human mast cell tryptase. J. Immunol. 130:2352-2356.

26. Mandle, R. J., R. W. Colman, and A. P. Kaplan. 1976. Identification of prekallikrein and high molecular weight kininogen as a complex in human plasma. Proc. Natl. Acad. Sci. USA. 73:41794183.

27. Jacobsen, S. 1966. Separation of two different substrates for plasma kinin-forming enzymes. Nature (Lond.). 210:98-99.

28. Mygind, N., B. Weeke, and S. Ullman. 1975. Quantitative determinations of immunoglobulins in nasal secretions. Int. Arch. Allergy Appl. Immunol. 49:99-107.

29. Mason, B., and A. A. Miles. 1962. Globulin permeability factors without kininogenase activity. Nature (Lond.). 196:587-588.

30. Webster, M. E., and J. V. Pierce. 1963. The nature of the kallidins released from human plasma by kallikreins and other enzymes. Ann. NY Acad. Sci. 104:91-107. 